\title{
No acute effects of grape juice on appetite, implicit memory and mood
}

\author{
Sara J. Hendrickson and Richard D. Mattes \\ Department of Foods and Nutrition, Purdue University, West Lafayette, IN, USA
}

\section{Abstract}

Background: Animal experiments document effects of grape juice on cognitive performance and motor skills, and observational studies in humans suggest an inverse association between flavonoid intake and cognitive decline. These effects may be related to the antioxidant properties of polyphenols. Juice consumption and flavonoid intake may also affect appetite.

Objective: To study the acute effects of grape juice consumption on appetite, mood and implicit memory during a time of increased lethargy - the post-lunch dip.

Design: Thirty-five participants with a mean age of 26 years who smoked a mean of 11 cigarettes/day for 8 years were included in the study. It included a practice session and two treatment sessions. All sessions involved consumption of grape juice or an energy-matched placebo with lunch followed by assessments of mood, implicit memory, appetite and food intake.

Results: Mood decreased over time for both treatments, but there were no differences after lunch between grape juice and placebo for any measure.

Conclusion: This study did not document any acute effects of grape juice consumption on mood, implicit memory, appetite or food intake in smokers.

Keywords: grape juice; polyphenols; appetite; implicit memory; post-lunch dip

Received: II April 2008; Revised: 14 July 2008; Accepted: 21 November 2008; Published: 19 December 2008

$\mathrm{C}$ erebral blood flow $(\mathrm{CBF})$ is fundamental to brain function (1). Enhanced brain blood flow to meet task demands correlates with performance on measures of cognitive function (2). Cardiovascular disease risk factors, such as elevated plasma homocysteine and blood pressure, are negatively associated with processing capacity/speed and executive function, respectively, in older men (3). Additionally, in old age, atherosclerosis is associated with a hastened decline in memory (4). Likewise, cognitive function (5) and processing capacity/executive function (6) are negatively associated with arterial stiffness, which, in turn, is associated with endothelial function (7). Thus, factors that improve endothelial function and/or other mechanisms affecting CBF may improve cognitive function as well.

Ginkgo biloba, cocoa and grape products are dietary components that may affect CBF. Ginkgo biloba increases $\mathrm{CBF}(8)$ and can improve aspects of attention and memory in young, healthy participants (9). Although a causal role has not been established, part of its proposed neuroprotective effect may be due to its flavonoid fraction (10). Flavanols, a flavonoid subgroup found in cocoa, may provide similar effects. Consumption of a flavanol-rich cocoa drink acutely increased circulating nitric oxide and endothelium-dependent dilation in participants who smoke (11). The dilation could lead to increased CBF as noted following consumption of a high-flavanol cocoa drink in healthy young participants (12). Grape products reduce platelet aggregation (13), which could lower blood viscosity and therefore increase blood flow. Red grape polyphenol extract increased flow-mediated dilatation in men with coronary heart disease (14), suggesting grape juice (GJ) may also affect CBF. Thus, studying the effects of GJ on cognition is warranted. This research would complement studies indicating improved cognitive performance and motor skills in aging rats from GJ (15), greater flavonoid intake being associated with higher baseline cognitive performance and reduced decline over time in older humans (16), as well as studies supporting an inverse association between consumption of fruit/vegetable juice and Alzheimer's disease risk in humans (17).

While GJ (or its polyphenols) may provide certain health benefits, the full range of its influence must be considered since consumption of some energy-yielding 
beverages may encourage positive energy balance by evoking a weak compensatory dietary response (18). However, in contrast to this literature, grape-seed extract tablets reduced energy intake in subjects with energy requirements greater than or equal to $7.5 \mathrm{MJ} /$ day (19). Further, rats fed a high fat diet along with red wine had lower energy intake and did not gain as much weight as control rats consuming a high fat diet (20). This study, therefore, explored the effects of GJ on both cognition and appetite.

\section{Materials and methods}

Participants were recruited through public announcements. Eligibility requirements included body mass index $>18.5 \mathrm{~kg} / \mathrm{m}^{2}$ (mean $24.8 \pm \mathrm{SD} 4.5 \mathrm{~kg} / \mathrm{m}^{2}$ ), age $18-50$ years $(26 \pm 7.5)$, no current use of antioxidant dietary supplements or a willingness to refrain from their use three weeks prior to and during the study, and selfreported smoking of $\geq 3$ cigarettes/day (11.0 \pm 5.1$)$ for $\geq 1$ year $(8.0 \pm 6.3)$. Smoking status was confirmed by a saliva sample analyzed for cotinine by Salimetrics, LLC, State College, PA $(189.3 \pm 121.0 \mathrm{ng} / \mathrm{ml})$. Plasma ascorbic acid, determined by the method of Lykkesfeldt et al. (21), was $50.5 \pm 28.2 \mu \mathrm{M}$, which was between the $59.1 \mu \mathrm{M}$ detected in passive smokers and the $40.3 \mu \mathrm{M}$ detected in persons smoking $\geq 15$ cigarettes per day (22).

The study was double-blind, within-subject and placebo-controlled. Every participant completed three sessions. The first session was a practice session, and data were not recorded. Half of the participants consumed GJ in the first experimental session and placebo in the second; the other half followed the opposite order. Most participants' three sessions were on the same day of the week over the course of three consecutive weeks.

Each session consisted of two visits to the laboratory a lunch visit and a diet recall visit the following day. The treatments were $10 \mathrm{ml} / \mathrm{kg}$ body weight of a placebo beverage or $100 \%$ Concord GJ containing 2,100 mg/l total phenolics as gallic acid equivalents (beverages and data supplied by Welch Foods, Inc., Concord, MA). The placebo was formulated to match Concord GJ for energy, fructose and glucose content, acidity, taste, color and aroma. Participants were instructed to eat a similar breakfast on each treatment day (which participants reported during the following day's diet recall) and not to consume any food or energy-containing beverage within three hours of arrival at the laboratory. Participants were also instructed not to consume any additional GJ or red wine for the entire day.

For the lunch visit, participants arrived at the laboratory between 12:00 and 13:00 hours on weekdays and completed a Profile of Mood States questionnaire (POMS) (Multi-Health Systems Inc., North Tonawanda, NY) and an affect grid (AG) (23). Next, participants completed the first of four word fragment completion
(WFC) tasks (see description below), followed by an appetite assessment. Appetite ratings were provided on a personal digital assistant (PDA) programmed to record marks made by participants on visual analog scales. Next, participants had up to 30 minutes to consume a lunch of as much Kraft Easy Mac (Kraft Foods Inc., Northfield, IL) as desired, one 99 g Hunt's Snack Pack Butterscotch Pudding cup (ConAgra Foods Inc., Omaha, NE), and the treatment beverage. After lunch, participants provided appetite ratings and completed a second POMS and AG. Then, they performed a second WFC task. Immediately afterward, another AG was completed, followed by a third WFC task and another AG. Finally, a fourth and final WFC task was completed, followed by a third set of appetite ratings, the final POMS and the final AG. During all WFC tasks, participants wore noise-reducing headphones to reduce auditory distractions (Maxell Noise Cancellation Headphone, Maxell Corporation of America, Fair Lawn, NJ).

After leaving the laboratory, participants provided hourly appetite ratings on the PDAs and continued recording their food intake for the remainder of the day. The following day, participants returned their PDA to the laboratory and participated in a computer-aided 24-hour diet recall interview with Nutrition Data System for Research software (NDS-R, 2005 and NDSR, 2006 Nutrition Coordinating Center, University of Minnesota, Minneapolis, MN).

Due to the nature of a within-subject study design and the desire not to arouse participants out of the post-lunch dip by administering a novel test of mental function, changes in mental ability were assessed in a covert manner on a repetitive task using a modified test of implicit memory. This test involved a WFC task where participants were shown a fragment (e.g. _ha_ad_) and were asked to fill in the missing spaces to make a word (e.g. charade). For assessing implicit memory, this task usually involves prior presentation of the unfragmented words in a long list (24). Later, fragments of those words are presented along with fragments of new words, and participants are asked to complete the fragments (24). The increased likelihood of participants completing the fragments with previously studied words is considered priming (25). In the present study, this method was altered to avoid changing tasks during the sessions. Only a set of word fragments was presented for priming. Unfragmented words were not provided. Due to its monotonous nature, this task was not only unlikely to arouse participants out of the post-lunch dip, but may have augmented its development.

Participants were given 20 minutes to complete each of the four, 24-word tasks. Fragments were considered incomplete if there was no response, if the response was spelled incorrectly or if the response was not a primed word. Six of the fragments in the first task were repeated 
in the final task to serve as the test of implicit memory, and only the proportion of these words completed correctly is reported. Thirty-six words were used as priming words, divided into six sets of six words that were counter-balanced among the thirty-six participants and three sessions in order to avoid confounding by word set. As there were not enough fragments in the source used (26), some additional distraction fragments were introduced by the researchers.

An affective post-lunch dip state was expected to be induced and maintained after 30 minutes of performing the WFC tasks based on a previous study attempting to induce the post-lunch dip through a letter cancellation task (27). The final fragment completion task was performed by participants approximately 47-67 minutes after finishing lunch, so they were within the expected post-lunch dip time both by the previous study and by indications that the post-lunch dip occurs one hour after beginning lunch (28). Further, because debate exists regarding whether the post-lunch dip is related to consuming lunch or circadian rhythms, the study was designed to administer the final fragment completion task between approximately 13:30 and 14:45 hours, depending on when participants arrived and how quickly they consumed lunch. Thus, the timing of this study was designed so that participants completed the fourth fragment completion task both at the appropriate time of day and period of time after consuming lunch for the postlunch dip to occur. Further, seeking effects between 45 and 65 minutes after consuming the juice coincides with other research documenting acute effects of grape polyphenol consumption. These acute effects include improved flow mediated dilatation from dealcoholized red wine as compared to regular red wine in coronary artery disease patients (29) and increased serum total antioxidant capacity (30). Finally, as several participants requested, but were denied, a cigarette smoking break upon completion of lunch, participants may have experienced further deficits due to forced smoking abstinence during this timeframe. Thus, participants were tested with the final WFC task just as the GJ polyphenols were expected to begin inducing physiological effects, and the post-lunch dip, smoking abstinence and tedium of a repetitive task were likely to impair their ability/motivation.

Participants were compensated financially for participation, and the study protocol was approved by the Purdue University Institutional Review Board using signed informed consent.

\section{Statistical analysis}

Data were analyzed using SPSS 14.0 for Windows (SPSS, Inc., Chicago, IL). Upper and lower outliers were removed if they were greater or lower than the value of the third or first quartile plus one and one-half times the interquartile range, respectively (31). The proportion correct on WFC tasks (by-person and by-word) and the post-lunch dip affective state were analyzed for a main effect of time and a time-by-juice interaction using repeated measures analysis of variance. Tests of withinsubject effects used the Huynh-Feldt correction. Food intake and appetite ratings were analyzed for differences between treatments using the Wilcoxon Signed Ranks Test. Two-tailed $p$-values less than 0.05 were considered significant.

\section{Results}

Thirty-six participants completed the protocol. Thirtythree participants provided saliva samples, and one individual had a cotinine concentration below the 7-13 $\mathrm{ng} / \mathrm{ml}$ cutoff recommended for identifying smokers (32) so was not included in further analyses. Thus, the final sample included 18 females and 17 males.

WFC task results are shown in Table 1. In both the "by-person" and "by-word" analyses, the proportion correct for word fragments before lunch was unexpectedly and significantly higher in the GJ treatment: $F(1,27)=7.01, p=0.01$ and $F(1,35)=4.84, p=0.04$, respectively (Table 1$)$. There was no time-by-juice interaction or main effect of time.

For the AG (Table 2), there were no time-by-juice interactions, but there was a significant overall effect of

Table 1. Mean (standard deviation) for repeated word fragment completion (WFC), hunger, fullness and energy intake

\begin{tabular}{|c|c|c|c|c|}
\hline Outcome measure & Grape juice & $n$ & Placebo & $n$ \\
\hline \multicolumn{5}{|c|}{ WFC by person (\% correct) } \\
\hline Before lunch & $58(17)^{*}$ & 28 & $48(22)$ & 28 \\
\hline After lunch & $59(15)$ & 28 & $54(23)$ & 28 \\
\hline \multicolumn{5}{|c|}{ WFC by item (\% correct) } \\
\hline Before lunch & $61(29)^{*}$ & 36 & $52(27)$ & 36 \\
\hline After lunch & $62(30)$ & 36 & $58(26)$ & 36 \\
\hline \multicolumn{5}{|l|}{ Mean hunger (mm) } \\
\hline Mean & $20.6($ (II.I) & 35 & $17.7(9.8)$ & 35 \\
\hline Peak & $44.2(18.9)$ & 35 & $38.9(18.6)$ & 35 \\
\hline Nadir & $3.4(3.1)$ & 32 & $4.1(4.4)$ & 34 \\
\hline \multicolumn{5}{|l|}{ Mean fullness (mm) } \\
\hline Mean & $53.5(12.8)$ & 33 & $58.4(16.1)$ & 34 \\
\hline Peak & $82.1(15.5)$ & 34 & $83.9(13.2)$ & 34 \\
\hline Nadir & $23.8(17.9)$ & 34 & $31.5(22.6)$ & 35 \\
\hline \multicolumn{5}{|c|}{ Energy intake (kilocalories) } \\
\hline Before lunch & $211(233)$ & 35 & $228(236)$ & 35 \\
\hline Lunch & $854(192)$ & 35 & $840(193)$ & 35 \\
\hline After lunch & I494 (655) & 35 & $|2| \mid(475)$ & 32 \\
\hline 24-hour & $2559(686)$ & 35 & $2282(4 I I)$ & 32 \\
\hline
\end{tabular}

*Significantly higher than placebo $(p<0.05)$. 
Table 2. Affect grid ratings, mean (standard error) time effects

\begin{tabular}{lcc}
\hline Affect grid number & $\begin{array}{c}\text { Pleasure-displeasure } \\
(n=33)\end{array}$ & $\begin{array}{c}\text { Arousal sleepiness } \\
(n=34)\end{array}$ \\
\hline 1 & $6.6(0.2)$ & $5.4(0.2)$ \\
2 & $6.2(0.2)$ & $5.1(0.2)$ \\
3 & $6.2(0.2)$ & $4.7(0.2)^{*}$ \\
4 & $6.0(0.2)^{*}$ & $4.3(0.3)^{*}$ \\
5 & $6.1(0.2)^{*}$ & $4.1(0.3)^{*}$
\end{tabular}

*Significantly different from baseline measurement $(p<0.05)$.

time for both pleasure-displeasure, $F(3.6,115.0)=2.89$, $p=0.03$, and arousal-sleepiness, $F(3.0,97.9)=12.52$, $p<0.01$. For pleasure-displeasure, the final two postlunch measurements were significantly lower than baseline. For arousal-sleepiness, the final three measurements were significantly lower than baseline.

For the POMS questionnaire (Table 3), there were no time-by-juice interactions. However, time had an overall significant effect for confusion-bewilderment, $F(1.9$, $59.0)=4.14, \quad p=0.02 ;$ fatigue-inertia, $F(1.4,41.2)=$ $15.37, p<0.01$; total mood disturbance (TMD), $F(1.7$, $50.6)=10.03, p<0.01$; and vigor-activity, $F(2.0,66.0)=$ $34.78, p<0.01$. For confusion-bewilderment, fatigueinertia and TMD, the second rating was not different from baseline, but the third rating was significantly higher than baseline. For vigor-activity, both the second and third ratings were significantly lower than baseline.

For the appetite ratings, during the free-living period after lunch, at least $50 \%$ of participants provided entries hourly from 15:00 to 21:00 hours. The data from those seven time points, along with the two in-laboratory entries provided after lunch, were used to determine mean, peak and nadir ratings. There were no significant differences in hunger, fullness or energy intake (Table 1).

\section{Discussion}

For the cognitive component, this study mirrored a recommendation from research on cocoa beverages to test participants in a state of fatigue. This augments

Table 3. Profile of mood state (POMS), ratings mean (standard error) time effects

\begin{tabular}{lcccc}
\hline & $\begin{array}{c}\text { Confusion- } \\
\text { bewilderment } \\
(n=32)\end{array}$ & $\begin{array}{c}\text { Fatigue- } \\
\text { inertia } \\
(n=30)\end{array}$ & $\begin{array}{c}\text { Total mood } \\
\text { disturbance } \\
(n=30)\end{array}$ & $\begin{array}{c}\text { Vigor- } \\
\text { activity } \\
(n=34)\end{array}$ \\
\hline 1 & $41.3(0.8)$ & $39.3(0.7)$ & $45.7(0.7)$ & $42.3(1.2)$ \\
2 & $41.5(0.8)$ & $38.8(0.7)$ & $45.9(0.7)$ & $38.7(1.2)^{*}$ \\
3 & $42.7(1.0)^{*}$ & $42.0(0.9)^{*}$ & $47.6(0.7)^{*}$ & $36.4(1.1)^{*}$ \\
\hline
\end{tabular}

*Significantly different from baseline measurement $(p<0.05)$. identification of treatment effects (12) as it allows for measurement of revival of function or protection from a performance decline (1). This study did so through use of the post-lunch dip, which is the deterioration in mental function that often occurs after consuming the mid-day meal (33). All measures of mood were significantly modified over time in the direction expected to indicate the presence of a post-lunch dip by the time of the final mood assessment. However, the lack of time-by-juice interactions indicates beverage type had no effect on subjective feelings of the post-lunch dip.

To further enhance measurement sensitivity, participants with likely increased risk for oxidative stress were selected for this study. This was accomplished by recruiting individuals who smoked at least three cigarettes daily for at least one year (34). This sub-group may be more likely to benefit from antioxidant treatment and have been used previously in research on grape products as a model of oxidative stress (35). Smokers also tend to have low antioxidant vitamin intake (36).

As WFC performance was not significantly changed over time for either treatment, it appears GJ does not affect implicit memory and/or that performance on individual words is too stable to be affected. Also, WFC rates before lunch were consistently higher in the GJ treatment, which may have predisposed the results toward non-significance or favoring the placebo as there was less freedom to improve over time in the GJ treatment.

This study failed to detect effects of acute GJ consumption by smokers on measures of appetite/food intake, mood and implicit memory. Chronic dosing may have yielded different results. Future research should explore the effects of a chronic dosing regimen on a wider variety of cognitive tasks, physiological measures and possibly a larger and different subset of participants (e.g. nonsmokers).

\section{Conflict of interest and funding}

Treatment beverages supplied by Welch Foods, Inc., Concord, MA. WFC consultation provided by Dr. James S. Nairne, PhD, Purdue University. Ascorbic acid analysis assistance provided by Dr. John R. Burgess, Purdue University. Work supported by Grant No. 5 P50 AT00477 from the National Center for Complimentary and Alternative Medicine (NCAAM) and the office of Dietary Supplements and a HATCH grant from the USDA \#IND 084055H. Richard Mattes is engaged in other research supported by Welch's.

\section{References}

1. Dinges DF. Cocoa flavanols, cerebral blood flow, cognition, and health: going forward. J Cardiovasc Pharmacol 2006; 47: S223-5.

2. Boles Ponto LL, Magnotta VA, Moser DJ, Duff KM, Schultz SK. Global cerebral blood flow in relation to cognitive 
performance and reserve in subjects with mild memory deficits. Mol Imaging Biol 2006; 8: 363-72.

3. Aleman A, Muller M, de Haan EHF, van der Schouw YT. Vascular risk factors and cognitive function in a sample of independently living men. Neurobiol Aging 2005; 26: 485-90.

4. Vinkers DJ, Stek ML, van der Mast RC, de Craen AJM, Le Cessie S, Jolles J, et al. Generalized atherosclerosis, cognitive decline, and depressive symptoms in old age. Neurology 2005; 65: 107-12.

5. Fukuhara M, Matsumura K, Ansai T, Takata Y, Sonoki K, Akifusa $\mathrm{S}$, et al. Prediction of cognitive function by arterial stiffness in the very elderly. Circ J 2006; 70: 756-61.

6. Muller M, Grobbee DE, Aleman A, Bots M, van der Schouw YT. Cardiovascular disease and cognitive performance in middle-aged and elderly men. Atherosclerosis 2007; 190: 143-9.

7. Nigam A, Mitchell GF, Lambert J, Tardif J-C. Relation between conduit vessel stiffness (assessed by tonometry) and endothelial function (assessed by flow-mediated dilatation) in patients with and without coronary heart disease. Am J Cardiol 2003; 92: 395-9.

8. Ahlemeyer B, Krieglstein J. Neuroprotective effects of Ginkgo biloba extract. Cell Mol Life Sci 2003; 60: 1779-92.

9. Kennedy DO, Scholey AB, Wesnes KA. The dose-dependent cognitive effects of acute administration of Ginkgo biloba to healthy young volunteers. Psychopharmacology 2000; 151: 416-23.

10. Smith PF, Maclennan K, Darlington CL. The neuroprotective properties of the Ginkgo biloba leaf: a review of the possible relationship to platelet-activating factor (PAF). J Ethnopharmacol 1996; 50: 131-9.

11. Heiss C, Kleinbongard P, Dejam A, Perre S, Schroeter H, Sies H, et al. Acute consumption of flavanol-rich cocoa and the reversal of endothelial dysfunction in smokers. J Am Coll Cardiol 2005; 46: 1276-83.

12. Francis ST, Head K, Morris PG, Macdonald IA. The effect of flavanol-rich cocoa on the fMRI response to a cognitive task in healthy young people. J Cardiovasc Pharmacol 2006; 47: S21520 .

13. Shanmuganayagam D, Beahm MR, Osman HE, Krueger CG, Reed JD, Folts JD. Grape seed and grape skin extracts elicit a greater antiplatelet effect when used in combination than when used individually in dogs and humans. J Nutr 2002; 132: 3592-8.

14. Lekakis J, Rallidis LS, Andreadou I, Vamvakou G, Kazantzoglou G, Magiatis P, et al. Polyphenolic compounds from red grapes acutely improve endothelial function in patients with coronary heart disease. Eur J Cardiovasc Prev Rehabil 2005; 12: 596-600.

15. Shukitt-Hale B, Carey A, Simon L, Mark DA, Joseph JA. Effects of Concord grape juice on cognitive and motor deficits in aging. Nutrition 2006; 22: 295-302.

16. Letenneur L, Proust-Lima C, Le Gouge A, Dartigues JF, Barberger-Gateau P. Flavonoid intake and cognitive decline over a 10-year period. Am J Epidemiol 2007; 165: 1364-71.

17. Dai Q, Borenstein AR, Wu Y, Jackson JC, Larson EB. Fruit and vegetable juices and Alzheimer's disease: the Kame Project. Am J Med 2006; 119: 751-9.

18. Mattes R. Fluid calories and energy balance: the good, the bad, and the uncertain. Physiol Behav 2006; 89: 66-70.

19. Vogels N, Nijs IMT, Westerterp-Plantenga MS. The effect of grape-seed extract on $24 \mathrm{~h}$ energy intake in humans. Eur J Clin Nutr 2004; 58: 667-73.

20. Bargallo MV, Grau AA, Fernandez-Larrea JD, Anguiano GP, Segarra MCB, Rovira MJS, et al. Moderate red-wine consumption partially prevents body weight gain in rats fed a hyperlipidic diet. J Nutr Biochem 2006; 17: 139-42.
21. Lykkesfeldt J, Loft S, Poulsen HE. Determination of ascorbic acid and dehydroascorbic acid in plasma by high-performance liquid chromatography with coulometric detection - are they reliable biomarkers of oxidative stress? Anal Biochem 1995; 229: 329-35.

22. Dietrich M, Block G, Norkus EP, Hudes M, Traber MG, Cross $\mathrm{CE}$, et al. Smoking and exposure to environmental tobacco smoke decrease some plasma antioxidants and increase \{gamma\}-tocopherol in vivo after adjustment for dietary antioxidant intakes. Am J Clin Nutr 2003; 77: 160-6.

23. Russell JA, Weiss A, Mendelsohn GA. Affect grid: a single-item scale of pleasure and arousal. J Person Soc Psychol 1989; 57: 493-502.

24. Roediger III HL, Geraci L. Implicit memory tasks in cognitive research. In: Wenzel A and Rubin DC, eds. Cognitive methods and their application to clinical research. Washington, DC: American Psychological Association; 2005: 129-51.

25. Schacter DL. Implicit memory: history and current status. J Exp Psychol/Learn Mem Cogn 1987; 13: 501-18.

26. MacLeod CM, Kampe KE. Word frequency effects on recall, recognition, and word fragment completion tests. J Exp Psychol/ Learn Mem Cogn 1996; 22: 132-42.

27. Mattes RD, Pawlik MK. Effects of Ginkgo biloba on alertness and chemosensory function in healthy adults. Hum Psychopharmacol: Clin Exp 2004; 19: 81-90.

28. Craig A. Acute effects of meals on perceptual and cognitive efficiency. Nutr Rev 1986; 44: 163-71.

29. Karatzi K, Papamichael C, Aznaouridis K, Karatzis E, Lekakis J, Matsouka C, et al. Constituents of red wine other than alcohol improve endothelial function in patients with coronary artery disease. Coron Artery Dis 2004; 15: 485-90.

30. Day AP, Kemp HJ, Bolton C, Hartog M, Stansbie D. Effect of concentrated red grape juice consumption on serum antioxidant capacity and low-density lipoprotein oxidation. Ann Nutr Metab 1997; 41: 353-7.

31. Samuels ML, Witmer JA. Statistics for the life sciences, 3rd ed. Upper Saddle River, NJ: Prentice Hall, Pearson Education, Inc.; 2003.

32. Etter J-F, Due TV, Perneger TV. Saliva cotinine levels in smokers and nonsmokers. Am J Epidemiol 2000; 151: 251-58.

33. Kanarek R. Psychological effects of snacks and altered meal frequency. Br J Nutr 1997; 77: S105-20.

34. Zhou JF, Yan XF, Guo FZ, Sun NY, Qian ZJ, Ding DY. Effects of cigarette smoking and smoking cessation on plasma constituents and enzyme activities related to oxidative stress. Biomed Environ Sci 2000; 13: 44-55.

35. Vigna GB, Costantini F, Aldini G, Carini M, Catapano A, Schena F, et al. Effect of a standardized grape seed extract on low-density lipoprotein susceptibility to oxidation in heavy smokers. Metabolism 2003; 52: 1250-7.

36. Dallongeville J, Marecaux N, Fruchart J-C, Amouyel P. Cigarette smoking is associated with unhealthy patterns of nutrient intake: a meta-analysis. J Nutr 1998; 128: 1450-7.

\section{Richard D. Mattes}

Department of Foods and Nutrition

Purdue University

700 West State Street

West Lafayette, IN 47907, USA

Tel: + I 7654940662

Fax: + I 7654940674

E-mail: mattes@purdue.edu 